\title{
Comparative analysis of molecular signatures suggests the use of gabapentin for the management of endometriosis-associated pain
}

This article was published in the following Dove Press journal: Journal of Pain Research

Brice Bellessort,' Anne Bachelot, 1,2 Virginie Grouthier, ${ }^{2}$ Camille De Lombares,' Nicolas Narboux-Neme,' Paolo Garagnani, ${ }^{3,4}$ Chiara Pirazzini, ${ }^{3,4}$ Simonetta Astigiano, ${ }^{5}$ Luca Mastracci, ${ }^{6,7}$ Anastasia Fontaine,' Gladys Alfama, Evelyne Duvernois-Berthet,' Giovanni Levi'

'Evolution of Endocrine Regulations, Department AVIV, National Museum of Natural History, Paris, France; ${ }^{2}$ AP-HP, Department of Endocrinology and Reproductive Medicine, Reference Center for Rare Endocrine Diseases, Pitié-Salpêtrière Hospital, UPMC, Paris, France; ${ }^{3}$ Department of Experimental, Diagnostic and Specialty Medicine, Alma Mater Studiorum, University of Bologna, Bologna, Italy; ${ }^{4}$ Interdepartmental Center "L. Galvani", University of Bologna, Bologna, Italy; ${ }^{5}$ Department of Integrated Oncological Therapies, San Martino Hospital, Genova, Italy; 'Department of Experimental Medicine and Center of Excellence for Biomedical Research, University of Genoa, Genova, Italy; ' ${ }^{7}$ Division of Anatomic Pathology, Department of Surgical Science and Integrated Diagnostics, University of Genoa, Genova, Italy

\section{Video abstract}

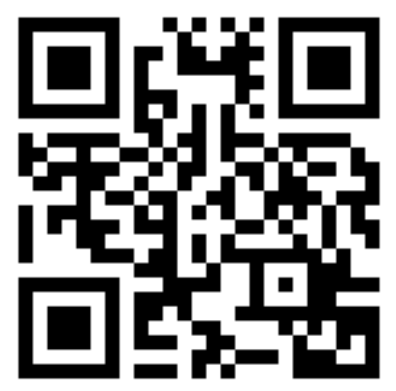

Point your SmartPhone at the code above. If you have a QR code reader the video abstract will appear. Or use: http://youtu.be/KHskcrdazLc

Correspondence: Giovanni Levi

CNRS UMR 7221, Évolution des Régulations

Endocriniennes, Muséum National d'Histoire Naturelle,

7 Rue Cuvier, 7523I Paris, France

$\mathrm{Tel}+33$ । 4079362 ।

Fax +33 | 40793618

Email glevi@mnhn.fr
Background: It has been repetitively shown that the transcription factors $D L X 5$ and $D L X 6$ are drastically downregulated in endometriotic lesions when compared with eutopic endometrium. These findings suggest that regulatory cascades involving DLX5/6 might be at the origin of endometriosis symptoms such as chronic pelvic pain (CPP). We have shown that inactivation of $D l x 5$ and $D l \times 5 / 6$ in the mouse uterus results in an endometrial phenotype reminiscent of endometriosis. Methods: We focused on genes that present a similar deregulation in endometriosis and in Dlx5/6-null mice in search of new endometriosis targets.

Results: We confirmed a strong reduction of $D L X 5$ expression in endometriosis implants. We identified a signature of 30 genes similarly deregulated in human endometriosis implants and in $D l x 5 / 6$-null mouse uteri, reinforcing the notion that the downregulation of $D l \times 5 / 6$ is an early event in the progress of endometriosis. $C A C N A 2 D 3$, a component of the $\alpha 2 \delta$ family of voltagedependent calcium channel complex, was strongly overexpressed both in mutant mouse uteri and in endometriosis implants, were also CACNA2D1 and CACNA2D2, other members of the $\alpha 2 \delta$ family involved in nociception, are upregulated.

Conclusion: Comparative analysis of gene expression signatures from endometriosis and mouse models showed that calcium channel subunits $\alpha 2 \delta$ involved in nociception can be targets for the treatment of endometriosis-associated pain. CACNA2D3 has been associated with pain sensitization and heat nociception in animal models. In patients, CACNA2D3 variants were associated with reduced sensitivity to acute noxious stimuli. As $\alpha 2 \delta$ s were targets of gabapentinoid analgesics, the results suggested the use of these drugs for the treatment of endometriosis-associated pain. Indeed, recent small-scale clinical studies have shown that gabapentin could be effective in women with CPP. The findings of this study reinforce the need for a large definitive trial.

Keywords: endometriosis, gabapentin, CACNA2D3, D1x5, pain

\section{Introduction}

Endometriosis is characterized by the growth of endometrial-like tissue consisting of glandular tissue and stroma outside the uterine cavity in localized and innervated implants. Remarkably, endometriosis lesions present histopathological and physiological responses that are similar to those of the endometrium. Endometriosis patients frequently experience various pain symptoms including chronic pelvic pain (CPP), dysmenorrhea, dyspareunia, and dyschezia, ${ }^{1}$ which often result in severe personal, social, and economic difficulties. Treatment of endometriosis-associated pain is difficult due to its poorly understood pathophysiology, complex clinical presentation, and natural history. Endometriosis is generally treated by surgical intervention, analgesic treatment, or hormonal treatment suppressing cyclic ovarian hormone production and 
reducing or eliminating menses. ${ }^{2}$ Current therapies present, however, limited efficacy on endometriosis pain, due to high rates of symptom recurrence, and significant side-effects. ${ }^{3,4}$

$D l x$ genes comprise a highly conserved family of homeobox genes. Dlx5 and Dlx6 (Dlx5/6) are expressed in GABAergic neurons of the brain including delta opioid receptor-positive GABAergic neurons that are involved in the regulation of chronic pain. ${ }^{5}$ Besides early roles in neuronogenesis and limb and craniofacial patterning during embryogenesis, ${ }^{6} \mathrm{Dl} x 5 / 6$ are also involved in the differentiation of steroidogenic tissues including Leydig cells in the testis $^{7}$ and theca and granulosa cells in the ovary. ${ }^{8} D L X 5$ and $D L X 6$ are expressed in glandular and lining epithelial cells of mouse and human endometrium ${ }^{9}$ where their expression is upregulated during the secretory phase ${ }^{10}$ of the cycle. By targeted inactivation of $D l x 5 / 6$ in the mouse uterus, we have demonstrated their central role for uterine adenogenesis and fertility. ${ }^{9}$

It has been repeatedly shown that the expression of $D L X 5$ and $D L X 6$ is strongly downregulated in endometriotic lesions compared to eutopic endometrium. ${ }^{1-14}$ Endometriosis implants and Dlx5/6 mutant mouse uteri have, therefore, in common a very low level of Dlx 5 expression. Furthermore, D1x5/6-null endometrial epithelia present a histological appearance reminiscent of that found in endometriosis implants with very few glands and a cuboidal lining epithelium that locally tends to desquamate. ${ }^{9}$ The histological and molecular similarities between endometriosis implants and Dlx5/6-null mouse uteri have prompted our suggestion that the downregulation of $D L X 5 / 6$ could be an early event in the progress of endometriosis and that inactivation of these genes in the mouse uterus could constitute a model of the human pathology. ${ }^{9}$

Here, we show that $D l \times 5 / 6$-null mouse uteri and ectopic human endometrium share 30 deregulated genes confirming the these mice can be considered a model of the disease; we suggest that these 30 shared genes are a new, more focused, molecular signature of endometriosis. We focus our attention on $C A C N A 2 D 3$, an overexpressed gene that has an evolutionary-conserved role in nociception. ${ }^{15} C A C N A 2 D 1$, $C A C N A 2 D 2$, and $C A C N A 2 D 3$ constitute the $\alpha 2 \delta$ gene family of glycosylphosphatidyl inositol (GPI)-anchored transmembrane voltage-gated calcium channel (VGCC)-associated subunits. During membrane depolarization, VGCCs permit $\mathrm{Ca}^{2}$ influx, contributing to cell depolarization and functioning as secondary messengers regulating key neuronal mechanisms including gene expression and neurotransmitter release. VGCCs are involved in generating neuropathic pain and are targets of gabapentinoids. ${ }^{16}$ Remarkably, $C A C N A 2 D 1$ to 3 are significantly increased in endometriosis implants ${ }^{12,13}$ and should therefore be considered as targets for the treatment of endometriotic pain.

\section{Methods}

\section{Endometrial biopsies}

A total of 37 ovarian endometriosis and 12 normal endometrium samples were selected from the formalin-fixed, paraffin-embedded tissue collection of the Division of Anatomic Pathology, Department of Surgical Science and Integrated Diagnostics, University of Genoa, Italy. All patients signed an informed consent under local institutional review board-approved protocols for research purposes dealing with the archived endometriosis tissues in the pathology department. Informed consents are available on request (from LM). The Regional Liguria Ethical Committee for medical research approved the use of archive human samples.

\section{Animals}

Procedures involving animals were conducted in accordance with the directives of the European Community (council directive 86/609) and the French Agriculture Ministry (council directive 87-848, October 19, 1987, permissions 00782 to GL). The project was reviewed and approved by the "Cuvier" ethical committee of the Muséum National d'Histoire Naturelle and transmitted to the French Ministry of Agriculture. Mice were housed in specific pathogen-free and light-, temperature- $\left(21^{\circ} \mathrm{C}\right)$, and humidity $(50-60 \%$ relative humidity)-controlled conditions. Food and water were

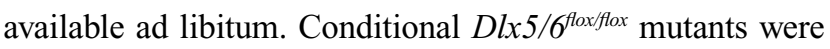
generated as described. ${ }^{9}$ To obtain $\mathrm{Pgr}^{\text {cre } /+} ; D l \times 5 / 6^{\text {flox } f l o x}$ mice, we crossed $P g r^{\text {cre/+ }} ; D l x 5 / 6^{f l o x /+}$ males with $D l x 5 / 6^{\text {floxflox }}$ females.

\section{Immunohistochemistry}

Fixed tissue samples were embedded in paraffin, and $5 \mathrm{~mm}$ thick sections were cut. Slides were dewaxed in xylol and rehydrated in ethanol solutions. Antigen exposure was achieved by submerging slides in sodium citrate $0.01 \mathrm{M}, \mathrm{pH} 6.0$ (Sigma-Aldrich Co., St Louis, MO, USA) and heating at $90^{\circ} \mathrm{C}$ with pressure in a 2100-Retriever device (Prestige Medical, Northridge CA, USA). Endogenous peroxidase activity was quenched by incubation with $3 \%$ (vol/vol) $\mathrm{H}_{2} \mathrm{O}_{2}$ in methanol (Merck Millipore, Billerica, MA, USA) or with peroxidaseblocking solution from Dako EnVision + System HRP kit (K4011; Agilent Technologies, Santa Clara, CA, USA) for 30 minutes. Tissue sections were then blocked with 5\% (wt/ vol) bovine serum albumin (BSA) (Sigma-Aldrich Co.) or 
$10 \%$ of fetal calf serum (FCS) in PBS at room temperature for 2 hours and then incubated with anti-DLX5 (NBP1-19547; Novus Biologicals, Littleton, CO, USA) polyclonal rabbit primary antibody at 1/200 dilution, or anti-CACNA2D3 at 1/250 dilution (NBP1-30557; Novus Biologicals), or anti-CBS at 1/400 dilution (1478-1-AP; Proteintech, Manchester, UK), or anti-LTBP2 at 1/250 dilution (17708-1-AP; Proteintech, UK), or anti-GJB3 at 1/50 dilution (12880-1-AP; Proteintech, UK), or anti-NOXA1 at 1/100 dilution (CUSABIO, Huston, TX, USA), or anti-KIAA1324 at 1/200 dilution (Thermo Fisher Scientific, Waltham, MA, USA) in PBS 5\% FCS overnight at $4^{\circ} \mathrm{C}$ in a humidified chamber. Normal rabbit IgGs were used as negative control. Next, slides were washed with PBS and detection of the primary antibody was achieved by using the Histostain-SP Broad Spectrum kit (Thermo Fisher Scientific) or goat antirabbit antibody linked to horseradish peroxidase (Dako EnVision + System HRP kit; K4011). Counterstain was done using the hematoxylin-stabilized solution 1/5 (RAL Diagnostics, Martillac, France) according to standard procedures. Images were visualized under an Olympus BX-51 microscope and captured using the Image-Pro Plus v.6.2 software (Media Cybernetics, Rockville, MD, USA).

\section{RNAseq}

RNA library preparation was carried out according to the Illumina TruSeq Stranded Total RNA Sample Prep protocol, following Ribo-Zero Gold Deplete procedure (Illumina, San Diego, CA, USA). From a total amount of $1.5 \mu \mathrm{g}$ of total RNA, both cytoplasmic RNA and mitochondrial ribosomal RNA (rRNA) were removed using specific biotinylated oligos and Ribo-Zero rRNA removal beads. RNA, purified

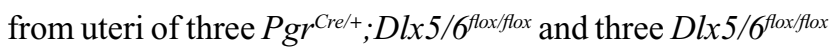
mice at PN25, was fragmented by the addition of divalent cations and the incubation at $94^{\circ} \mathrm{C}$ for 4 minutes. The first cDNA strand was synthesized using random primers and a reverse transcriptase. DNA polymerase I synthesized the second cDNA strand and generated blunt-ended ds cDNA, while RNase $\mathrm{H}$ removed the RNA template. An A nucleotide was added to the $3^{\prime}$ ends, followed by the ligation of multiple indexing adapters that are required for the pooling and the hybridization with the flow cell. These DNA products were purified, and only those having adapter molecules on both ends were enriched with PCR by using a Cocktail Primer that anneals specifically to the adapters. The obtained libraries were validated by Bioanalyzer (Agilent DNA 1000; Agilent Technologies) and pooled and sequenced using the Illumina HiSeq 2000 sequencing system. The global quality of each library was checked using the FastQC software (http://www.bioinformatics.babraham. ac.uk/projects/fastqc/). To reduce any PCR amplification bias, duplicated reads were removed before the duplicated reads before the mapping based on a $100 \%$ sequence identity between reads. Then, five nucleotides were trimmed at both $5^{\prime}$ and $3^{\prime}$ ends of all remnant reads using FASTXToolkit v0.0.14 (http://hannonlab.cshl.edu/fastx_toolkit/ index.html). Reads were mapped against the mouse mm10 genome (Version GRCm38.p3) using the TopHat2 software v2.0.10 17 with default parameters. Only uniquely mapped reads were kept and were counted on genes' exons using the HTSeq-count software v0.6.1p1 18 with "union" mode using the Mus musculus Ensembl annotation (release 79). Only coding and expressed genes were considered. " $R$ " package DESeq2 v1.8.0 ${ }^{19}$ was used for statistical analyses to determine differential gene expression levels (cutoff: adjusted $P$-value $\leq 0.1$ ). Samples' homogeneity was checked realizing a principal component analysis with " $R$ " package FactoMineR, following which, one mutant mouse was not considered for further analysis.

To identify mouse and human orthologs, we used the BioMart tool, provided by Ensembl, Heidelberg, Germany.

From 424 genes differentially expressed in $\mathrm{Pgr}^{\text {cre/t+ }}$; Dlx $5 / 6^{f l o x / f l o x}$ mice, we found 391 human orthologs, which have been used for comparison.

\section{Quantitative real-time PCR (qPCR)}

Total RNA was isolated from five mouse uterine samples per group using an RNeasy Mini Kit (Qiagen NV, Venlo, the Netherlands) according the manufacturer's instructions. On-Column Deoxyribonuclease I (Hoffman-La Roche Ltd., Basel, Switzerland) digestion was incorporated into an RNA isolation procedure to remove potential genomic DNA contamination. RNA concentration and the ratio of the absorbance at 260 and $280 \mathrm{~nm}$ were measured using a NanoDrop 2000 spectrophotometer (Thermo Fisher Scientific). Reverse transcription was carried out using 600 or $200 \mathrm{ng}$ total RNA and Primscript (Ozyme) reverse transcriptase to obtain cDNA. qPCR was performed using the QuantStudio 6 Flex Real-Time PCR System (Thermo Fisher Scientific). The PCR program consisted of $95^{\circ} \mathrm{C}$ for 10 minutes, 40 cycles $95^{\circ} \mathrm{C}$ for 15 seconds, and $60^{\circ} \mathrm{C}$ for 10 minutes. Relative gene expression was measured using SYBR Green mix gene expression assays (Thermo Fisher Scientific). Primers utilized are shown in Table 1. To measure the relative amount of PCR products, the Ct geometric mean of Sdha and Hprt1, was subtracted from the $\mathrm{Ct}$ of genes of interest to derive $\Delta \mathrm{Ct}$. The $\Delta \mathrm{Ct}$ of mutant animals was compared with $\Delta \mathrm{Ct}$ of control 
Table I PCR primers used in this study

\begin{tabular}{|c|c|c|c|}
\hline Genes & Forward primer & Reverse primer & Length (bp) \\
\hline NOXAI & CCGCTGGCTAGGATGTACTT & CAGCTGGAAATTGGCCACTC & 150 \\
\hline DMRTAI & CCCAACTTTCGAGGTTTTCCA & CCCAGAGAATGGTGATGAGTGTT & 126 \\
\hline KIAAI 324 & TAGACCTCCACAGTCAGTGGT & GCATTCTCTTCGATGGTGTAGG & 198 \\
\hline SLC27A2 & TCCTCCAAGATGTGCGGTACT & TAGGTGAGCGTCTCGTCTCG & 166 \\
\hline GABRP & ATGAGCTACAGCCTCTATTTGGC & ACCACCGAAATTGGGCCTG & 174 \\
\hline SAPCD2 & GGGCTCTTCTCAGTGTGACA & ACCTTCTTCAGCACTTGTGC & 106 \\
\hline PLCHI & GGCTCGTTCGCCTCTTTTAC & CTGCCCTCGGTGACTTTGTAG & 118 \\
\hline GJB3 & GCTCCAAGACCTATTGAGTGGC & GCCTGGTGTTACAGTCAAAGTC & 155 \\
\hline DPP6 & CAGAGGCAAAGTGGATAAGCA & TCAACATTCCGCAGTATGACAC & 76 \\
\hline OSCPI & TGCTTTACGTCCTAGACCAGC & GGTTTGAACAGTTCGTCCATGA & 124 \\
\hline JAZFI & GCCGAGAACAGGAATCTCTGA & GTAAGGCTGCCACTGCTATGT & 118 \\
\hline CBS & GGGACAAGGATCGAGTCTGGA & AGCACTGTGTGATAATGTGGG & 100 \\
\hline NAIP5 & GCAGTCTCTCGAGGTCTCAG & CACACAGTTCTTTCAGGCCC & 89 \\
\hline EMB & TGAGGGCGATCCCACAGAT & CCGTCACTGAGATATTACAGCTC & 107 \\
\hline CLIP4 & AGAAGGTCGATGTAGCCCAC & TCCGCTACAGTTTTCGGTTC & 124 \\
\hline PIK3API & GTCCCGGATGCCTCTTTCTC & CACAAGTCATTTCCTGCCAGT & 241 \\
\hline LAPTM5 & ACATCGAATTGCCAGCGTAC & CAGCTGCAGTGTCATCAAGG & 80 \\
\hline CTGF & GGGCCTCTTCTGCGATTTC & ATCCAGGCAAGTGCATTGGTA & $|5|$ \\
\hline NAIP2 & TGTGCTCTCAGTCCTTCCTG & AAGCACAGTCATGAGAGGCT & 184 \\
\hline CACNA2D3 & AAGAAATCGACGGTCTCCAAC & GGTCATTGGGGGCTAAGATGAA & 237 \\
\hline LTBP2 & AACAGCACCAACCACTGTATC & CCTGGCATTCTGAGGGTCAAA & 159 \\
\hline TNS3 & ACATGCACTTCACCAACGTC & CTGAGAAACTGCACGTACCG & 130 \\
\hline RAB20 & GCAGTGGCGTTCCTTCAAC & GGAGCCCAGACCATGAAACT & 70 \\
\hline SYTL2 & TCAAAAAGCCGACCAAGAACC & AGGCACTGTGGAAATTCCTGG & 61 \\
\hline LBH & CTGCTCTGACTATCTGAGATCGG & CGGTCAAAGTCTGATGGGTCC & 135 \\
\hline $\mathrm{ENCI}$ & CTGTTTCATAAGTCCTCCTACGC & CACCACTGAACATGGCTTCG & 169 \\
\hline MANBA & GACTGTCCCCGGCTATGTG & AATTCCGTGCTGTAGGTCCA & 126 \\
\hline GALM & CTGCACGATCACTGCTCTG & CACTGCTCCAAAGTAGGGCTG & 115 \\
\hline
\end{tabular}

animals, and the difference was assigned as $\Delta \Delta \mathrm{Ct}$. The fold change between two samples was then calculated as $2^{-\Delta \Delta C t}$.

\section{Statistical analysis}

The Mann-Whitney unpaired test was conducted using Prism (GraphPad Software, Inc., La Jolla, CA, USA) to calculate the differences between groups of five independent measurements. All values are expressed as mean $\pm \mathrm{SEM}$ of combined data from replicate experiments. Values $P<0.05$ were considered statistically significant.

\section{Results \\ DLX5 distribution in normal endometrium and in endometriosis}

First, we analyzed the distribution of DLX5 in normal endometrium from various stages of the menstrual cycle by immunohistochemistry (Figure $1 \mathrm{~A}-\mathrm{B}^{\prime}$ ). DLX5 protein is prevalently present in endometrial glandular and luminal epithelial cells. The intracellular distribution of DLX5 varies at different phases of the cycle: during the proliferative phase,
DLX5 is detected both in the nucleus and in the cytoplasm (Figure 1A and $\mathrm{A}^{\prime}$ ), while in the secretory phase, DLX5 is predominantly present in the cytoplasm (Figure $1 \mathrm{~B}$ and $\mathrm{B}^{\prime}$ ) where it displays a polarized distribution mostly toward the luminal and basal aspects of the cells.

Most endometriotic lesions (Figure $1 \mathrm{C}-\mathrm{D}^{\prime}$ ) present very few glands with a very large lumen covered by an atypical noncolumnar epithelium, which does not present a uniform phenotype even between different epithelial regions from the same patient (eg, the two facing epithelia in Figure 1D'). In certain regions, epithelial cells are large and noncohesive (Figure $1 \mathrm{C}^{\prime}$ and $\mathrm{D}^{\prime}$ ), while in other territories, they display a flattened phenotype with very little cytoplasm (Figure 1D' and $\mathrm{E}^{\prime}$ ); cells are almost invariably ciliated. The intensity of anti-DLX5 staining is generally low or at the limit of detection (Figure 1C-D'), but occasionally (Figure 1E and $\mathrm{E}^{\prime}$ ), a clear signal can be detected in the cytoplasm and in perinuclear regions, while nuclei are mostly negative; frequently, epithelia are constituted by a mixture of DLX5-positive and DLX5-negative (arrows in Figure 1E') cells. 

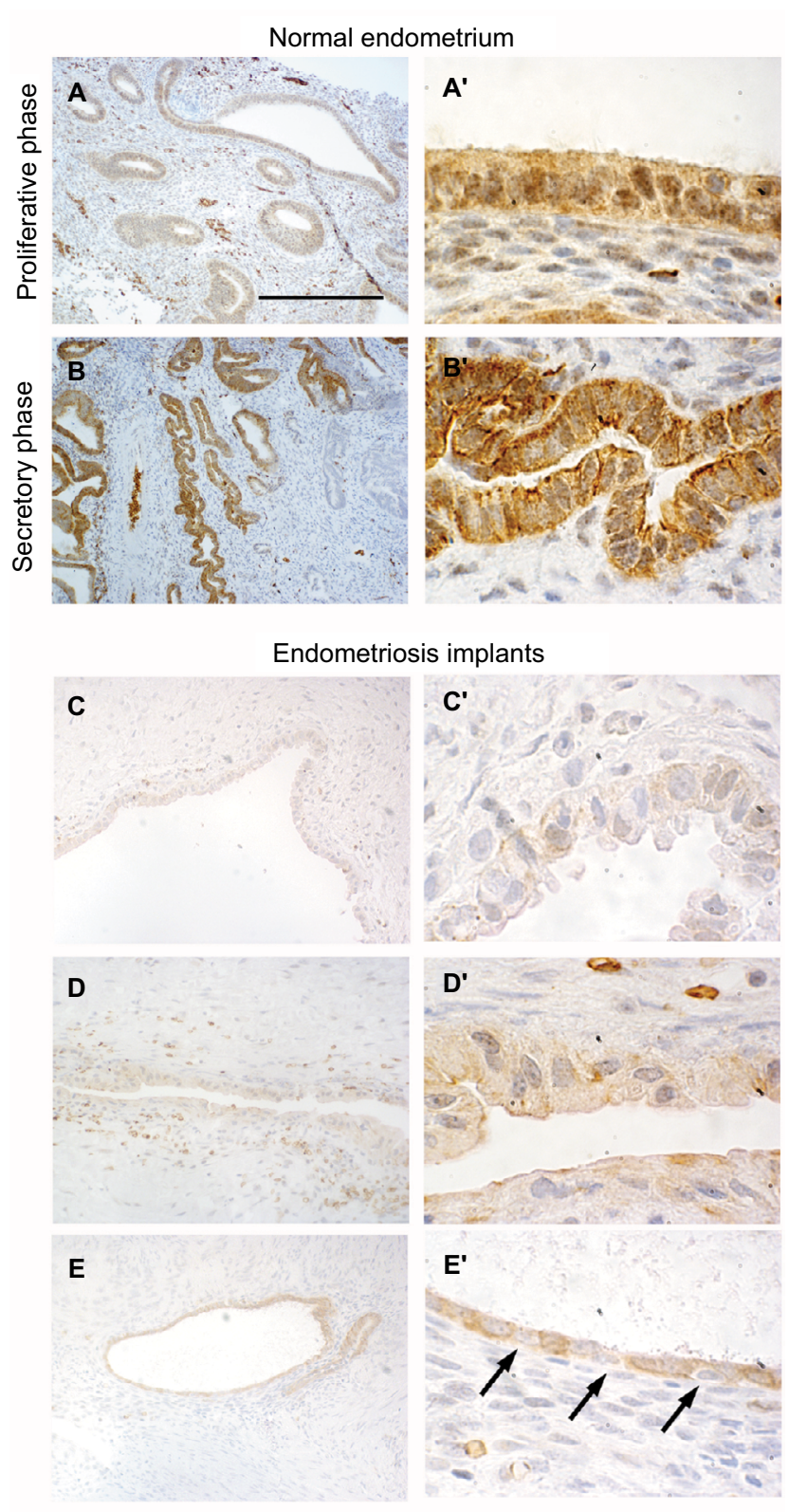

Figure I Immunohistolocalization of DLX5 in normal endometrium and ovarian endometriosis.

Notes: In healthy endometrium, DLX5 protein is detected in glandular epithelia $\left(\mathbf{A}, \mathbf{A}^{\prime}, \mathbf{B}\right.$, and $\left.\mathbf{B}^{\prime}\right)$. During the proliferative phase ( $\mathbf{A}$ and $\left.\mathbf{A}^{\prime}\right)$, the protein is present both in epithelial nuclei and in the cytoplasm. In contrast, during the secretory phase (B and $\mathbf{B}^{\prime}$ ) DLX5 presents a polarized distribution in the cytoplasm and is virtually absent from the nuclei. In ovarian endometriosis implants $\left(\mathbf{C}-\mathbf{E}^{\prime}\right)$, the expression of DLX5 is more variable. Most of the implants present very few glands with a very large lumen and a thin cuboidal epithelia, which is in general constituted by a mixture of DLX5-positive and DLX5-negative (arrows in $\left.\mathbf{E}^{\prime}\right)$ cells. Scale bar: ( $\mathbf{A}$ and B) $250 \mu \mathrm{m}$; (C-E) $125 \mu \mathrm{m}$; and $\left(\mathbf{A}^{\prime}, \mathbf{B}^{\prime}, \mathbf{C}^{\prime}, \mathbf{D}^{\prime}\right.$, and $\left.\mathbf{E}^{\prime}\right) 25 \mu \mathrm{m}$.

\section{A shared genetic signature between Dlx5/6-null mouse uteri and endometriosis implants}

$D L X 5$ and $D L X 6$ are between the most downregulated genes in endometriosis implants, ${ }^{11-13}$ and their inactivation in the mouse uterus results in an endometriosis-like phenotype. ${ }^{9}$ To better investigate the apparent similarity between endometriotic implants and mouse Dlx5/6-null uteri, we have compared the RNAseq profiles of genes deregulated in the mutant mouse uterus with those reported in four independent studies in which the expression profile of endometriosis implants was compared with that of eutopic endometrium. ${ }^{12-14,20}$ Hawkins et al ${ }^{12}$ described 2083 deregulated genes in endometriotic lesions; among this set of genes, 997 genes are underexpressed and 1086 genes are overexpressed. Several of these genes were also identified in the three other studies, which we have considered, ${ }^{13,14,20}$ providing a shared database to identify potential therapeutic targets. Comparison of Dlx5/6-mouse mutant uterus RNAseq dataset with the human datasets permits identifying 30 genes that present a common deregulation in the mouse and in endometriotic lesions: 13 downregulated and 17 upregulated genes (Table 2). If indeed the downregulation of $D l x 5 / 6$ is an early event in endometriosis progression, this set of 30 genes could well constitute a more focused molecular signature of endometriosis implants (Figure 2).

\section{Validation by qPCR}

In a first round of validation, we analyzed, by qPCR, the effect of Dlx5/6 inactivation on the expression levels of 28 of the 30 genes identified by RNAseq comparison (Figure 3A and B). In all cases, the trend of variation occurred in the direction predicted by RNAseq either up (Figure 3A) or down (Figure 3B); however, due to high individual variability, the variation reached a level of significance for only 12 genes. Between the upregulated genes, the expression of CACNA2D3 and CBS was, respectively, increased six and seven times. CACNA2D3 together with CACNA2D1 and CACNA2D2 constitutes the family of $\alpha 2 \delta$ VGCC subunits involved in nociception. In Dlx5/6-null mouse uteri, the expression levels of CACNA2D1 and $C A C N A 2 D 2$ were not significantly changed after RNAseq analysis and were not further analyzed.

Remarkably, the expression of each of these three genes is significantly increased in endometriosis implants (Data Set Records GDS2835 and GDS3975). ${ }^{12,13}$ Interestingly, the cystathionine- $\beta$-synthase (CBS) gene has been already shown to be hypomethylated and upregulated in endometriosis lesions. ${ }^{21}$

\section{Altered pattern of gene expression in human endometriosis}

To validate the upregulation in endometriosis of the most significantly affected genes, we performed immunohistolocalization on section from normal endometrium and 
Table 2 Genes commonly deregulated after D/x5/6 inactivation in the mouse uterus and in human endometriosis implants

\begin{tabular}{|c|c|c|c|c|c|c|c|}
\hline \multirow[t]{2}{*}{ Gene } & \multicolumn{2}{|c|}{$\begin{array}{l}\text { Comparison of normal and } \\
\text { DIx5/6-null mouse uterus }\end{array}$} & \multicolumn{5}{|c|}{$\begin{array}{l}\text { Comparison of normal human endometrium } \\
\text { and endometriosis implants }\end{array}$} \\
\hline & $\begin{array}{l}\text { Log } 2 \text { fold } \\
\text { change }\end{array}$ & $P$-value & $\begin{array}{l}\text { Log2 fold } \\
\text { change } \\
\text { Hawkins }\end{array}$ & $\begin{array}{l}P \text {-value } \\
\text { Hawkins }\end{array}$ & Hever & Hull & Eyster \\
\hline \multicolumn{8}{|l|}{ Down } \\
\hline DLX5 & \multicolumn{2}{|c|}{ Absent (targeted deletion) } & $-8,434$ & I.804E-04 & Down & & Down \\
\hline NOXAI & -2.25 & 3.5IE-07 & -1.816 & $2.012 \mathrm{E}-03$ & Down & & \\
\hline DMRTAI & -2.11 & $2.65 \mathrm{E}-06$ & -2.158 & $2.628 \mathrm{E}-04$ & & & \\
\hline KIAAI 324 & -2.07 & $1.96 \mathrm{E}-07$ & -19.062 & I. $180 \mathrm{E}-05$ & Down & Down & Down \\
\hline SLC27A2 & -1.91 & I. I 4E-05 & -1.982 & $5.256 \mathrm{E}-03$ & Down & Down & \\
\hline GABRP & -1.81 & $5.56 \mathrm{E}-06$ & -22.036 & 2.67IE-05 & Down & & \\
\hline SAPCD2 & -1.55 & 5.04E-04 & -2.155 & 6. $115 \mathrm{E}-03$ & Down & & \\
\hline CENPW & -1.36 & $6.92 \mathrm{E}-04$ & -1.905 & I.224E-04 & Down & & \\
\hline $\mathrm{PLCHI}$ & -1.32 & 4. $14 \mathrm{E}-04$ & -3.082 & $5.696 \mathrm{E}-04$ & Down & Down & \\
\hline GJB3 & -1.23 & $2.97 \mathrm{E}-03$ & -1.835 & $5.448 \mathrm{E}-0 \mathrm{I}$ & Down & & \\
\hline DPP6 & -1.21 & $2.80 \mathrm{E}-03$ & -2.917 & 2. $170 \mathrm{E}-04$ & Down & & \\
\hline OSCPI & -0.95 & $3.24 \mathrm{E}-03$ & -1.714 & I.668E-03 & & & Down \\
\hline JAZFI & -0.88 & $3.09 \mathrm{E}-03$ & -2.719 & $2.028 \mathrm{E}-06$ & Down & & \\
\hline \multicolumn{8}{|l|}{ Up } \\
\hline CBS & 3.09 & 5.77E-27 & 2.491 & $3.844 \mathrm{E}-03$ & $U_{p}$ & & Up \\
\hline NAIP5 & 2.44 & $1.69 \mathrm{E}-10$ & 1.884 & 3.807E-04 & & & \\
\hline EMB & 1.76 & $4.38 \mathrm{E}-08$ & 1.532 & $5.658 \mathrm{E}-03$ & & & \\
\hline CLIP4 & 1.52 & $1.76 \mathrm{E}-08$ & 2.612 & $2.198 \mathrm{E}-04$ & $U_{p}$ & & \\
\hline PIK3API & 1.48 & $8.44 \mathrm{E}-06$ & 2.566 & 2.857E-03 & Up & & \\
\hline LAPTM5 & 1.45 & $8.85 \mathrm{E}-05$ & 3.092 & $7.976 \mathrm{E}-03$ & $U_{p}$ & & Up \\
\hline CTGF & 1.29 & 3.5 IE- 04 & 1.582 & 7.149E-03 & & & Up \\
\hline NAIP2 & 1.19 & $1.04 \mathrm{E}-03$ & 1.884 & 3.807E-04 & & & \\
\hline CACNA2D3 & 1.19 & $2.6 I E-03$ & 2.066 & $2.469 \mathrm{E}-06$ & $U_{p}$ & & \\
\hline LTBP2 & 1.14 & $3.16 \mathrm{E}-03$ & 4.344 & $9.850 \mathrm{E}-05$ & $U_{p}$ & Up & Up \\
\hline TNS3 & 1.12 & $1.22 \mathrm{E}-05$ & 1.583 & $3.773 \mathrm{E}-03$ & Up & Up & \\
\hline RAB20 & 1.08 & $3.26 \mathrm{E}-04$ & 1.827 & $9.691 \mathrm{E}-03$ & & & \\
\hline SYTL2 & 0.97 & $1.97 \mathrm{E}-03$ & 1.788 & $8.826 \mathrm{E}-04$ & Up & & \\
\hline LBH & 0.97 & $1.26 \mathrm{E}-03$ & 2.029 & $1.615 \mathrm{E}-03$ & & & Up \\
\hline $\mathrm{ENCl}$ & 0.91 & $2.39 \mathrm{E}-03$ & 2.919 & $3.286 \mathrm{E}-03$ & Up & & \\
\hline MANBA & 0.83 & $1.98 \mathrm{E}-03$ & 2.011 & $2.363 \mathrm{E}-03$ & Up & & \\
\hline GALM & 0.76 & $2.27 \mathrm{E}-03$ & 1.680 & $1.019 \mathrm{E}-03$ & Up & & \\
\hline
\end{tabular}

endometriosis implants. CACNA2D3, CBS, and LTBP2 (Figure 4) immunodetection signal was dramatically increased in endometriotic lesions. CACNA2D3 was virtually undetectable on normal endometrium sections (Figure 4A and $\mathrm{B}$ ) and presented a high level of expression in endometriosis epithelia and smooth muscle cells (Figure $4 \mathrm{~A}^{\prime}$ and $\mathrm{B}^{\prime}$ ). In the epithelia, CACNA2D3 immunodetection was predominantly associated to membranes and cytoplasm (arrows in Figure $4 \mathrm{~B}^{\prime}$ ), while CBS and LTBP2 immunodetection (Figure $4 \mathrm{C}-\mathrm{D}^{\prime}$ ) was mostly nuclear in endometriosis implants.

\section{Discussion}

Morphological and genetic observations on mutant mouse uteri and on pathological specimens have prompted the suggestion that low $D L X 5$ and $D L X 6$ expressions could be associated with the progression of endometriosis. We have therefore proposed that Dlx5/6-null mouse uteri could be considered as a model of the disease permitting to get new insight on this pathology. ${ }^{9}$

Here, we have compared the gene expression signature of $D l x 5 / 6$-null mouse uteri with those obtained in four independent studies on endometriosis. ${ }^{12-14,20} \mathrm{Dl} \times 5 / 6$-null uteri and endometriosis implants share a set of 30 commonly deregulated genes supporting the hypothesis that downregulation of Dlx $5 / 6$ could be an early event in endometriosis progression. The 30 identified genes constitute a more "focused" genetic signature of the disease that has permitted potential therapeutic targets to be handpicked. We focused, in particular, on genes upregulated in endometriosis as they could be potential targets for therapeutic strategies. 
Current endometriosis therapies present limited efficacy, high rates of symptom recurrence, and significant side effects. ${ }^{3,4}$ For example, the use of GnRH agonists is

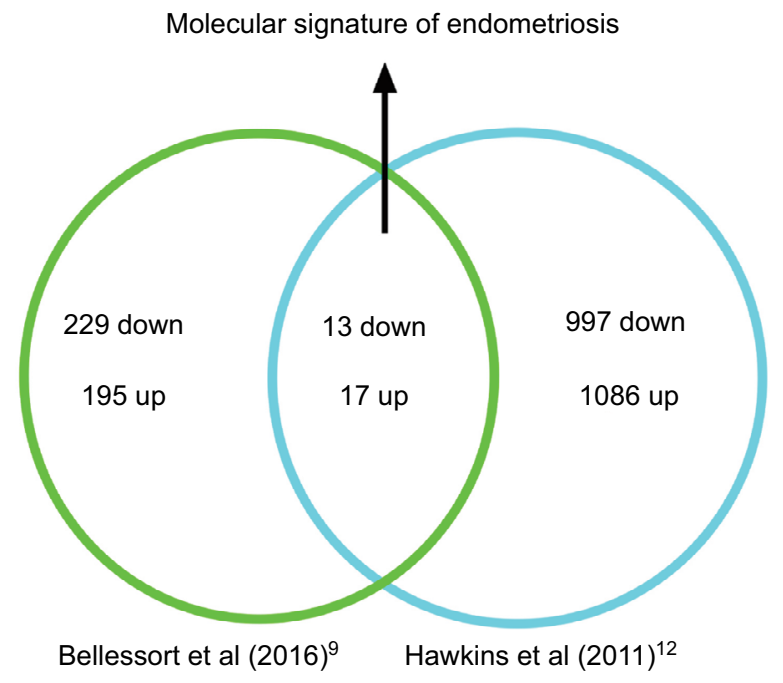

Figure 2 Molecular signature of endometriosis obtained comparing genes deregulated in D/x5/6 mutant mice uteri and in endometriosis lesions. associated with side effects such as menopausal symptoms and reduced bone mineral density, ${ }^{22}$ which restrict their use and demand add-back therapy. ${ }^{23}$ In addition, pain management is one of the major clinical challenges for the treatment of endometriosis. ${ }^{24}$ Endometriosis implants, although ectopic, are highly innervated. Peritoneal endometriosis lesions present a high density of sensory, cholinergic, and adrenergic nerve fibers that might be involved in the origin of pain. ${ }^{25,26}$ It has been proposed that a bidirectional relation exists between nerve fibers and endometriotic lesions with a two-way interaction between the implants and the central nervous system. ${ }^{27-29}$ The interplay between the peripheral and the central nervous system might be at the origin of individual differences in pain perception that can, in some patients, evolve independently from the disease. ${ }^{30}$ When pathological conditions cause damage to the nervous system, sensory activation thresholds are lowered with exaggerated pain perception even upon mild or absent painful stimulation.
A
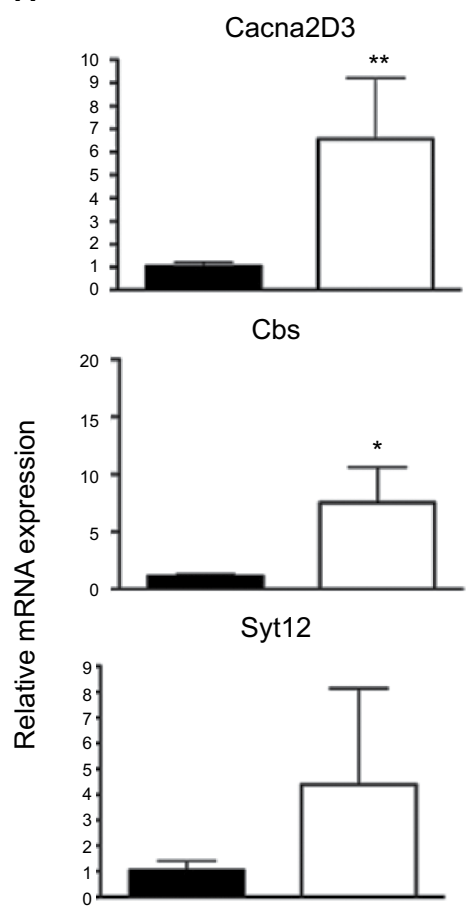

CLIP4

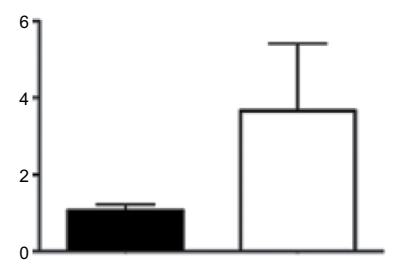

Rab20

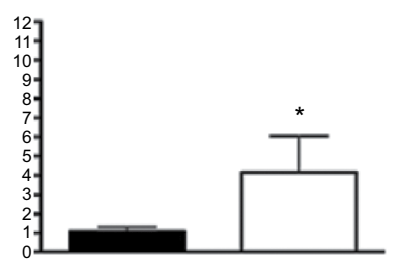

Galm
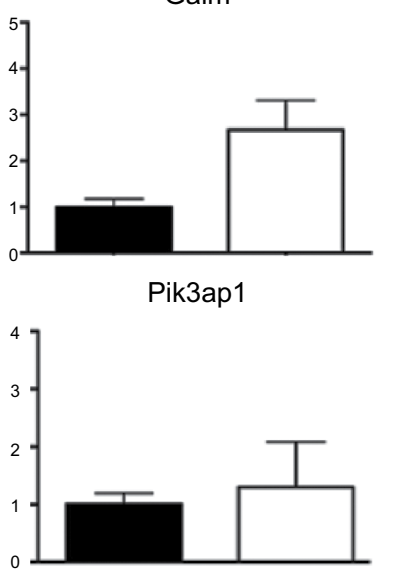

Ctgf

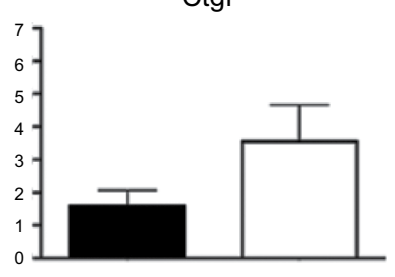

Ltbp2

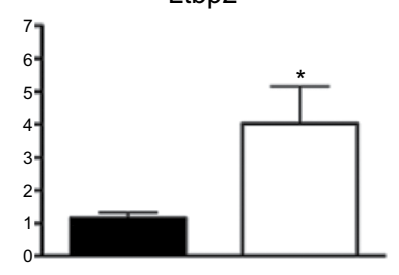

Laptm5

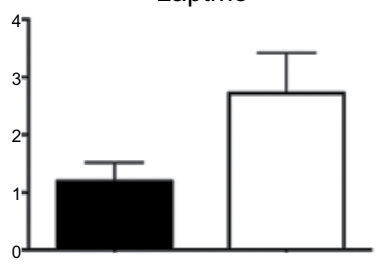

Naip2

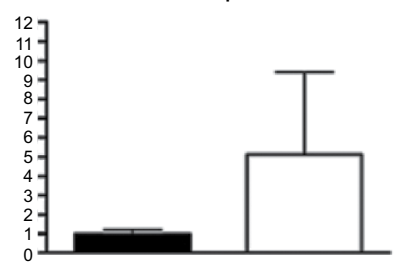

Enc1

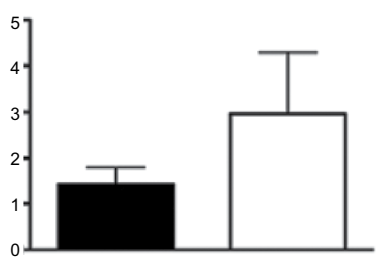

Emb

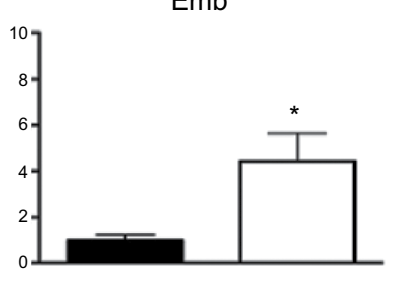

Lbh

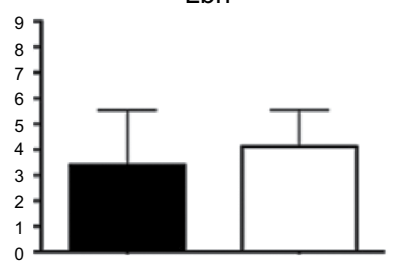

Manba

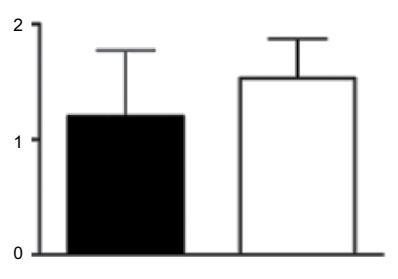

Tns3

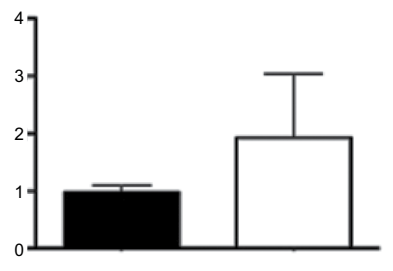

Figure 3 (Continued) 
B

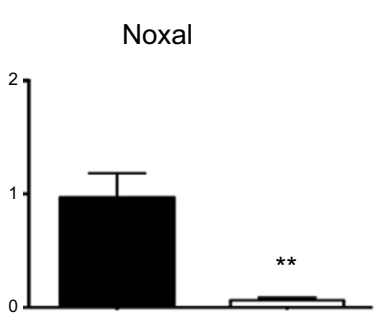

OScp1

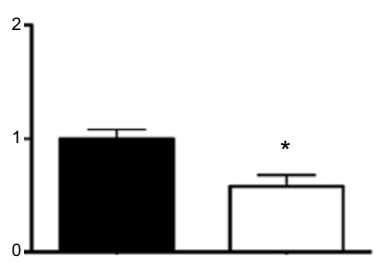

Gjb3

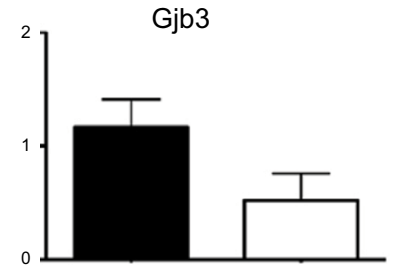

Gabrp

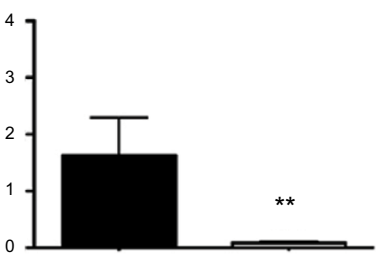

Dmrta1

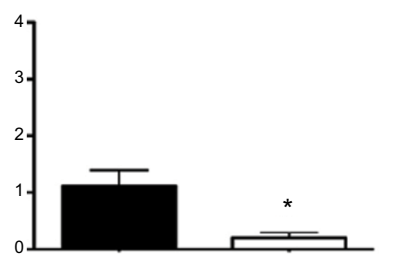

Sapcd2

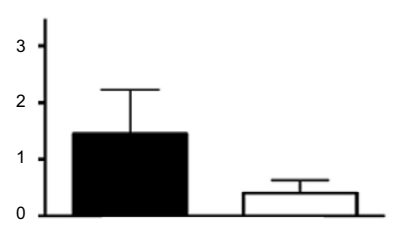

Kiaa1324

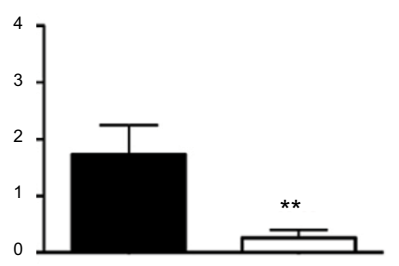

SIC27A2

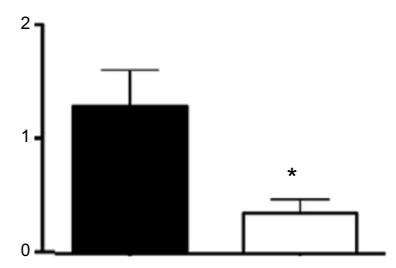

Nalp5

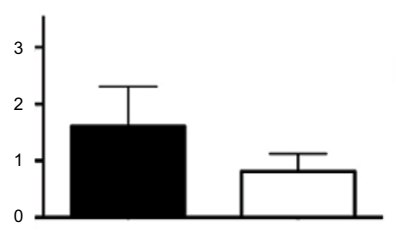

Plch1

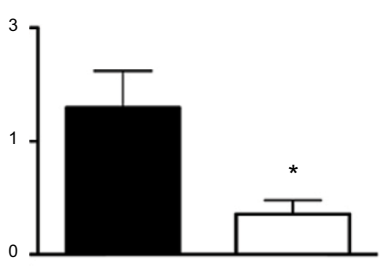

Jazf1

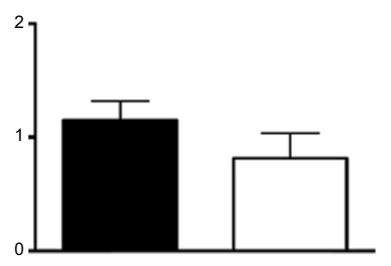

Dpp6

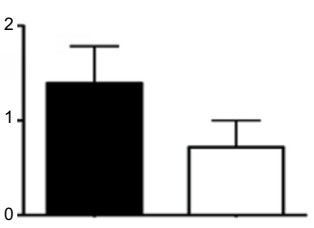

Figure 3 qPCR validation of genes upregulated after D/x5/6 invalidation in the mouse uterus.

Notes: (A) Relative mRNA expression in normal (black) and mutant (white bars) mouse uteri of upregulated genes belonging to our newly identified endometriosis signature. Reference gene: $S d h a .{ }^{*} P<0.05,{ }^{*} * P<0.01$, Mann and Whitney test analysis. Each group was constituted by five independent samples. (B) Relative mRNA expression in normal (black) and mutant (white bars) mouse uteri of downregulated genes identified as commonly deregulated genes belonging to our newly identified endometriosis signature (as shown in Table 2). Reference gene: Sdha. ${ }^{*} P<0.05$, ${ }^{*} * P<0.01$, Mann and Whitney test analysis. Each group was constituted by five independent samples. Abbreviation: qPCR, quantitative real-time PCR.

Between the 17 upregulated gene candidates, we focused specifically on CACNA2D3, member of the family of $\alpha 2 \delta$ VGCC subunits involved in nociception (Figure 5). CACNA2D1, CACNA2D2, CACNA2D3, and CACNA2D4 constitute the $\alpha 2 \delta$ family of VGCC subunits. ${ }^{16}$ VGCC are composed of $\alpha 1$ subunit proteins involved in pore formation $^{31}$ and many possible splice variants of the $\alpha 1$, $\beta$, and $\alpha 2 \delta$ subunits. Such a molecular diversity confers to these channels a variety of specific properties in different cell types and situations. VGCCs play a critical role in neuropathic pain development through the modulation of the release of excitatory neurotransmitters, ${ }^{32}$ calciumdependent enzyme activation, ${ }^{33}$ gene regulation, ${ }^{33-35}$ and short- and long-term plasticity changes. ${ }^{36-39}$ Abnormal regulation of VGCC subunits, such as $\alpha 2 \delta$ s, may contribute to pain signal transduction through several mechanisms including the induction of abnormal synaptogenesis..$^{40,41}$ Dysregulation of VGCCs and their subunits has been observed in pathological conditions, including nerve injuries and animal models of endometriosis; ${ }^{42}$ it is therefore possible that these channels contribute to the origin of endometriosis-associated pain.

Gabapentinoids, including gabapentin (Neurontin; Pfizer, Zürich, $\mathrm{CH}$ ) and pregabalin (Lyrica; Pfizer), are compounds used for the treatment of neuropathic pain..$^{43,44}$ As seen by binding of $\left[{ }^{3} \mathrm{H}\right]$ gabapentin to membranes from COS-7 cells transfected with $\alpha 2 \delta$ cDNA, gabapentinoids bind predominantly to $\alpha 2 \delta 1, \alpha 2 \delta 2$ subunits of VGCCs. ${ }^{45-47}$ However, an evolutionary conserved role in nociception has also been shown for $\alpha 2 \delta 3$, and SNP variants of this subunit are associated with reduced sensitivity to heat and chronic back pain. ${ }^{15}$

\section{Conclusion}

Analgesics, such as nonsteroidal anti-inflammatory drugs (NSAIDs) and gabapentin, are often used for pain relief despite limited evidence of their efficacy in endometriosis. ${ }^{4,48}$ 

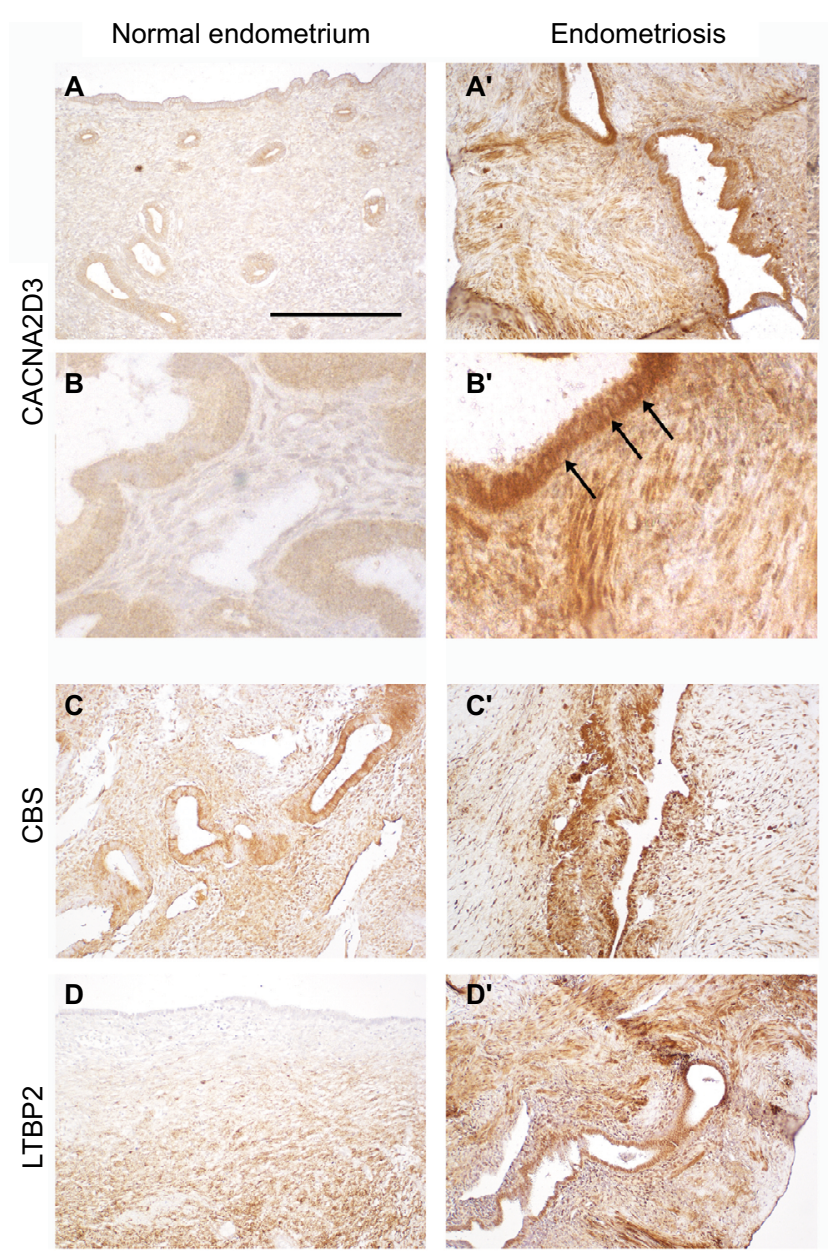

Figure 4 Immunolocalization of CACNA2D3, CBS, and LTBP2 in normal endometrium and in ovarian endometriosis implants.

Notes: Immunolocalization of CACNA2D3, CBS, and LTBP2 on sections from normal endometrium $(\mathbf{A}-\mathbf{C})$ and from endometriosis implants $\left(\mathbf{A}^{\prime}, \mathbf{B}^{\prime}\right.$, and $\left.\mathbf{C}^{\prime}\right)$. Arrows indicate CACNA2D3 distribution in epithelial cells of endometriosis implants. Scale bar: (A, $\mathbf{A}^{\prime}, \mathbf{C}, \mathbf{C}^{\prime}, \mathbf{D}$, and $\left.\mathbf{D}^{\prime}\right) 250 \mu \mathrm{m}$ and $\left(\mathbf{B}\right.$ and $\left.\mathbf{B}^{\prime}\right) 62.5 \mu \mathrm{m}$.
A recent pilot trial on 47 patients $^{49}$ and several clinical observations $\mathrm{s}^{48,50}$ have suggested that treatment with gabapentin alleviates significantly endometriotic pain; however, given the small size of the trials, uncertainty remains. ${ }^{43}$ Our findings provide evidence supporting a definitive evaluation of the efficacy of gabapentin in the management of endometriosis-associated pain.

\section{Acknowledgments}

We thank Dr Elisabeth Da Maia, Department of Pathology, Pitié Salpêtrière Hospital, for her kind help in endometrial and endometriosis section analysis. This research was partially supported by the EU Consortium HUMAN (EU-FP7-HEALTH-602757) to GL and by CNRS and MNHN general support to UMR7221. This study was supported in part by the French Centre National de la Recherche Scientifique, the French National Museum of Natural History, and the EU Consortium HUMAN (EU-FP7-HEALTH-602757).

\section{Author contributions}

$\mathrm{GL}, \mathrm{BB}, \mathrm{AB}, \mathrm{VG}, \mathrm{CDL}$, and NN-N conceptualized and designed the study. $\mathrm{BB}, \mathrm{AF}, \mathrm{GA}$, and $\mathrm{ED}-\mathrm{B}$ acquired the data. PG, CP, and ED-B generated and analyzed the RNAseq data. SA and LM collected the tissue and performed histological analysis. GL, BB, AB, and VG drafted the article. All authors discussed the results and commented on the article. All authors contributed toward data analysis, drafting and revising the paper and agree to be accountable for all aspects of the work.
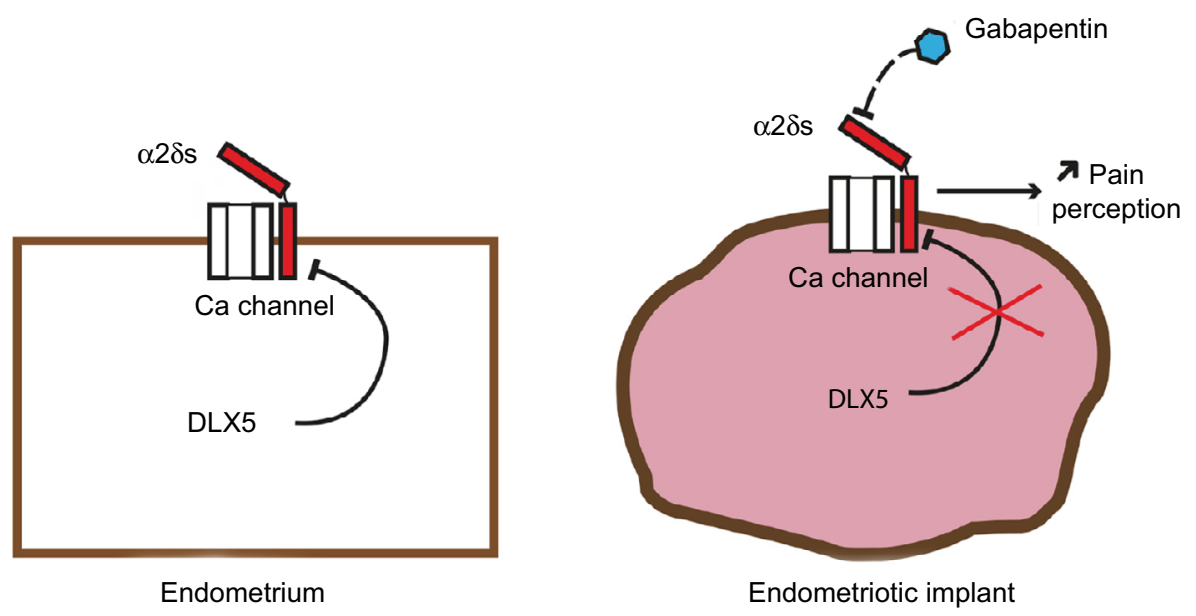

Figure 5 Summary diagram.

Note: The reduction of $D L X 5$ expression in endometriosis implants results in an increased expression of $\alpha 2 \delta$ subunits with a potential increase in neuropathic nociception. 


\section{Disclosure}

The authors report no conflicts of interest in this work.

\section{References}

1. Morotti M, Vincent K, Becker CM. Mechanisms of pain in endometriosis. Eur J Obstet Gynecol Reprod Biol. 2017;209:8-13.

2. Giudice LC. Clinical practice. Endometriosis. $N$ Engl J Med. 2010;362(25):2389-2398

3. Becker CM, Gattrell WT, Gude K, Singh SS. Reevaluating response and failure of medical treatment of endometriosis: a systematic review. Fertil Steril. 2017;108(1):125-136.

4. Dunselman GA, Vermeulen N, Becker C, et al; European Society of Human Reproduction and Embryology. ESHRE guideline: management of women with endometriosis. Hum Reprod. 2014;29(3):400-412.

5. Chu Sin Chung P, Keyworth HL, Martin-Garcia E, et al. A novel anxiogenic role for the delta opioid receptor expressed in GABAergic forebrain neurons. Biol Psychiatry. 2015;77(4):404-415.

6. Kraus P, Lufkin T. Dlx homeobox gene control of mammalian limb and craniofacial development. Am J Med Genet A. 2006;140(13): 1366-1374.

7. Nishida H, Miyagawa S, Vieux-Rochas M, et al. Positive regulation of steroidogenic acute regulatory protein gene expression through the interaction between Dlx and GATA-4 for testicular steroidogenesis. Endocrinology. 2008;149(5):2090-2097.

8. Bouhali K, Dipietromaria A, Fontaine A, et al. Allelic reduction of Dlx5 and Dlx6 results in early follicular depletion: a new mouse model of primary ovarian insufficiency. Hum Mol Genet. 2011;20(13): 2642-2650.

9. Bellessort B, Le Cardinal M, Bachelot A, et al. Dlx5 and Dlx6 control uterine adenogenesis during post-natal maturation: possible consequences for endometriosis. Hum Mol Genet. 2016;25(1): 97-108.

10. Talbi S, Hamilton AE, Vo KC, et al. Molecular phenotyping of human endometrium distinguishes menstrual cycle phases and underlying biological processes in normo-ovulatory women. Endocrinology. 2006;147(3):1097-1121.

11. Hu WP, Tay SK, Zhao Y. Endometriosis-specific genes identified by real-time reverse transcription-polymerase chain reaction expression profiling of endometriosis versus autologous uterine endometrium. J Clin Endocrinol Metab. 2006;91(1):228-238.

12. Hawkins SM, Creighton CJ, Han DY, et al. Functional microRNA involved in endometriosis. Mol Endocrinol. 2011;25(5):821-832.

13. Hever A, Roth RB, Hevezi P, et al. Human endometriosis is associated with plasma cells and overexpression of B lymphocyte stimulator. Proc Natl Acad Sci U S A. 2007;104(30):12451-12456.

14. Eyster KM, Boles AL, Brannian JD, Hansen KA. DNA microarray analysis of gene expression markers of endometriosis. Fertil Steril. 2002;77(1):38-42.

15. Neely GG, Hess A, Costigan M, et al. A genome-wide Drosophila screen for heat nociception identifies alpha2delta3 as an evolutionarily conserved pain gene. Cell. 2010;143(4):628-638.

16. Gong N, Park J, Luo ZD. Injury-induced maladaptation and dysregulation of calcium channel alpha2 delta subunit proteins and its contribution to neuropathic pain development. Br J Pharmacol. Epub 2017 Jun 23.

17. Kim D, Pertea G, Trapnell C, Pimentel H, Kelley R, Salzberg SL. TopHat2: accurate alignment of transcriptomes in the presence of insertions, deletions and gene fusions. Genome Biol. 2013;14(4):R36.

18. Anders S, Pyl PT, Huber W. HTSeq-a Python framework to work with high-throughput sequencing data. Bioinformatics. 2015;31(2):166-169.

19. Love MI, Huber W, Anders S. Moderated estimation of fold change and dispersion for RNA-seq data with DESeq2. Genome Biol. 2014;15(12):550.

20. Hull ML, Escareno CR, Godsland JM, et al. Endometrial-peritoneal interactions during endometriotic lesion establishment. Am J Pathol. 2008;173(3):700-715.
21. Borghese B, Barbaux S, Mondon F, et al. Research resource: genomewide profiling of methylated promoters in endometriosis reveals a subtelomeric location of hypermethylation. Mol Endocrinol. 2010;24(9):1872-1885.

22. Lee DY, Lee JY, Seo JW, Yoon BK, Choi D. Gonadotropin-releasing hormone agonist with add-back treatment is as effective and tolerable as dienogest in preventing pain recurrence after laparoscopic surgery for endometriosis. Arch Gynecol Obstet. 2016;294(6):1257-1263.

23. Lee DY, Park HG, Yoon BK, Choi D. Effects of different add-back regimens on hypoestrogenic problems by postoperative gonadotropinreleasing hormone agonist treatment in endometriosis. Obstet Gynecol Sci. 2016;59(1):32-38.

24. Berkley KJ, Rapkin AJ, Papka RE. The pains of endometriosis. Science. 2005;308(5728):1587-1589.

25. Tokushige N, Markham R, Russell P, Fraser IS. Nerve fibres in peritoneal endometriosis. Hum Reprod. 2006;21(11):3001-3007.

26. Tokushige N, Markham R, Russell P, Fraser IS. High density of small nerve fibres in the functional layer of the endometrium in women with endometriosis. Hum Reprod. 2006;21(3):782-787.

27. Morotti M, Vincent K, Brawn J, Zondervan KT, Becker CM. Peripheral changes in endometriosis-associated pain. Hum Reprod Update. 2014;20(5):717-736.

28. Miller EJ, Fraser IS. The importance of pelvic nerve fibers in endometriosis. Womens Health (Lond). 2015;11(5):611-618.

29. Yan D, Liu X, Guo SW. Nerve fibers and endometriotic lesions: partners in crime in inflicting pains in women with endometriosis. Eur J Obstet Gynecol Reprod Biol. 2017;209:14-24.

30. Stratton P, Berkley KJ. Chronic pelvic pain and endometriosis: translational evidence of the relationship and implications. Hum Reprod Update. 2011;17(3):327-346.

31. Dolphin AC. Voltage-gated calcium channels and their auxiliary subunits: physiology and pathophysiology and pharmacology. J Physiol. 2016;594(19):5369-5390.

32. Lee S. Pharmacological inhibition of voltage-gated $\mathrm{Ca}(2+)$ channels for chronic pain relief. Curr Neuropharmacol. 2013;11(6):606-620.

33. Park J, Luo ZD. Calcium channel functions in pain processing. Channels (Austin). 2010;4(6):510-517.

34. Perret D, Luo ZD. Targeting voltage-gated calcium channels for neuropathic pain management. Neurotherapeutics. 2009;6(4):679-692.

35. Wheeler DG, Groth RD, Ma H, et al. $\mathrm{Ca}(\mathrm{V}) 1$ and $\mathrm{Ca}(\mathrm{V}) 2$ channels engage distinct modes of $\mathrm{Ca}(2+)$ signaling to control CREB-dependent gene expression. Cell. 2012;149(5):1112-1124.

36. Liu XG, Zhou LJ. Long-term potentiation at spinal C-fiber synapses: a target for pathological pain. Curr Pharm Des. 2015;21(7):895-905.

37. Russo RE, Hounsgaard J. Short-term plasticity in turtle dorsal horn neurons mediated by L-type Ca2+ channels. Neuroscience. 1994;61(2):191-197.

38. Youn DH, Gerber G, Sather WA. Ionotropic glutamate receptors and voltage-gated $\mathrm{Ca}(2)(+)$ channels in long-term potentiation of spinal dorsal horn synapses and pain hypersensitivity. Neural Plast. 2013;2013:654257.

39. Naka A, Gruber-Schoffnegger D, Sandkuhler J. Non-Hebbian plasticity at C-fiber synapses in rat spinal cord lamina I neurons. Pain. 2013;154(8):1333-1342.

40. Eroglu C, Allen NJ, Susman MW, et al. Gabapentin receptor alpha2de1ta-1 is a neuronal thrombospondin receptor responsible for excitatory CNS synaptogenesis. Cell. 2009;139(2):380-392.

41. Park J, Yu YP, Zhou CY, et al. Central mechanisms mediating thrombospondin-4-induced pain states. J Biol Chem. 2016;291(25):13335-13348.

42. Wang Y, Zhang M, Xie F, et al. Upregulation of alpha(2)delta-1 calcium channel subunit in the spinal cord contributes to pelvic organ crosssensitization in a rat model of experimentally-induced endometriosis. Neurochem Res. 2015;40(6):1267-1273.

43. Moore RA, Wiffen PJ, Derry S, Toelle T, Rice AS. Gabapentin for chronic neuropathic pain and fibromyalgia in adults. Cochrane Database Syst Rev. 2014;4:CD007938. 
44. Zamponi GW, Striessnig J, Koschak A, Dolphin AC. The physiology, pathology, and pharmacology of voltage-gated calcium channels and their future therapeutic potential. Pharmacol Rev. 2015;67(4): 821-870.

45. Gong HC, Hang J, Kohler W, Li L, Su TZ. Tissue-specific expression and gabapentin-binding properties of calcium channel alpha2delta subunit subtypes. J Membr Biol. 2001;184(1):35-43.

46. Gee NS, Brown JP, Dissanayake VU, Offord J, Thurlow R, Woodruff GN. The novel anticonvulsant drug, gabapentin (Neurontin), binds to the alpha2delta subunit of a calcium channel. J Biol Chem. 1996;271(10):5768-5776.
47. Kukkar A, Bali A, Singh N, Jaggi AS. Implications and mechanism of action of gabapentin in neuropathic pain. Arch Pharm Res. 2013;36(3):237-251.

48. Speer LM, Mushkbar S, Erbele T. Chronic pelvic pain in women. Am Fam Physician. 2016;93(5):380-387.

49. Lewis SC, Bhattacharya S, Wu O, et al. Gabapentin for the management of chronic pelvic pain in women (GaPP1): a pilot randomised controlled trial. PLoS One. 2016;11(4):e0153037.

50. Sator-Katzenschlager SM, Scharbert G, Kress HG, et al. Chronic pelvic pain treated with gabapentin and amitriptyline: a randomized controlled pilot study. Wien Klin Wochenschr. 2005;117(21-22):761-768.
Journal of Pain Research

\section{Publish your work in this journal}

The Journal of Pain Research is an international, peer reviewed, open access, online journal that welcomes laboratory and clinical findings in the fields of pain research and the prevention and management of pain. Original research, reviews, symposium reports, hypothesis formation and commentaries are all considered for publication.

\section{Dovepress}

The manuscript management system is completely online and includes a very quick and fair peer-review system, which is all easy to use. Visit http://www.dovepress.com/testimonials.php to read real quotes from published authors. 